\title{
Disseminated nocardiosis caused by Nocardia otitidiscaviarum in an immunocompetent host: A case report and literature review
}

\author{
YANWEN JIANG, AIBEN HUANG and QIUHONG FANG \\ Department of Respiratory and Critical Care Medicine, Beijing Shijitan Hospital, \\ Capital Medical University, Beijing 100038, P.R. China
}

Received July 7, 2015; Accepted February 19, 2016

DOI: $10.3892 /$ etm.2016.3755

\begin{abstract}
The aim of the present study was to summarize the clinical characteristics of nocardiosis caused by Nocardia otitidiscaviarum in order to improve the knowledge of nocardiosis. A case of dissemination nocardiosis caused by $N$. otitidiscaviarum in an immunocompetent host is reported and the associated literature reviewed. Informed consent for publication of this case report was provided by the patient. The present patient was a young immunocompetent man suffering from disseminated nocardiosis induced by infection with $N$. otitidiscaviarum. Following a poor response to $\beta$-lactam antibiotic, a combination of sulfonamide with minocycline was administered, which successfully ameliorated the symptoms. Previous studies published in English were retrieved from PubMed with 'Nocardia otitidiscaviarum' used as the search keyword. A total of 23 articles were retrieved from the PubMed database, supporting the assertion that N. otitidiscaviarum is a rare Nocardia species. Among these 23 cases, there were 11 cases of lymphocutaneous (48\%), 5 of pulmonary (22\%), 2 of brain (9\%) and 1 of pyothorax (4\%) infection, and 4 cases of disseminated infections (17\%). Analysis of the immune state of these patients demonstrated that 9 were immunocompetent (39\%), 7 of whom had cutaneous infections $(30 \%)$ with a predominant history of trauma (6/7), and 14 were immunosuppressed, 9 of whom were treated with prednisolone. Microbiology and histopathology were necessary in all cases for definite diagnosis. Among the 13 cases who underwent drug susceptibility testing, 10 cases were sensitive to trimethoprim-sulfamethoxazole (TMP-SMX) and 12 cases were sensitive to aminoglycoside. In conclusion, although $N$. otitidiscaviarum is one of the less commonly isolated species of Nocardia, it is capable of inducing localized or disseminated infection, even in an immunocompetent
\end{abstract}

Correspondence to: Dr Qiuhong Fang, Department of Respiratory and Critical Care Medicine, Beijing Shijitan Hospital, Capital Medical University, 10 Tieyi Road, Beijing 100038, P.R. China E-mail: fanghxnk@126.com

Key words: Nocardia otitidiscaviarum, disseminative, lung, liver, subcutaneous, soft tissue, drug sensitivity host. The majority of cases respond well to TMP-SMX and aminoglycoside, but the therapeutic action of cephalosporin is weak. Identification of bacteria and drug sensitivity tests for Nocardia is critical for guiding clinical treatment.

\section{Introduction}

Nocardia spp. are gram-positive aerobic bacteria, which are commonly found in soil, water and air (1). Contaminated dust, soil and food can enter the body through the respiratory tract, gastrointestinal tract and skin and proceed to cause corresponding symptoms, such as coughing, expectoration, hemoptysis and skin abscesses, which are considered as nocardiosis (2). Nocardiosis is a vital, but often ignored, infectious disease in immunocompromised hosts, which is particularly serious in the absence of timely diagnosis and therapy. Nocardiosis can be caused by various Nocardia spp., including Nocardia asteroides, Nocardia brasiliensis, Nocardia cyriacigeorgica, Nocardia farcinica and Nocardia otitidiscaviarum (3). As compared with the other Nocardia spp., N. otitidiscaviarum appears to be rare $(3 \%)$ which was first recognized in samples taken from a Sumatran cavy or guinea pig with ear disease (3). The present case report describes a case of disseminated nocardiosis induced by infection with $N$. otitidiscaviarum in an immunocompetent host. Furthermore, in order to summarize the clinical features of this disease and improve understanding, a review of the existing literature of the disease induced by this species is also presented.

\section{Case report}

A 37 year-old Chinese man presented at Beijing Shijitan Hospital (Beijing, China) with an intermittent cough with little sputum, which had lasted 50 days, and a mass on the right side of his neck, which had first presented 1 month previously. The patient initially attributed the neck pain to a neck sprain, however the mass gradually increased in size over a period of 30 days. The patient complained of a 2-month history of unintentional weight loss ( $\geq 8 \mathrm{~kg}$ ) and mild pallor of the conjunctiva, skin and palms was detected. No fever, fatigue or night sweats had been experienced since the onset of disease. The patient had no past history of tuberculosis, diabetes mellitus or steroid therapy. The right-hand side of the patient's neck exhibited a 
marked swelling (12x8 cm; Fig. 1), the temperature of which was slightly elevated; however, no cervical or supraclavicular lymphadenopathy was detected. Following examination, percussion dullness was detected in the patient's chest from the first to sixth intercostal spaces on the right mid-clavicular line with a faint expiratory wheeze on the right. The heart rhythm of the patient was regular with no gallops or murmurs and abdominal examination raised no concerns.

Laboratory examination detected mild anemia with a hemoglobin concentration of $6.9 \mathrm{~g} / \mathrm{dl}$ (reference, $130-175 \mathrm{~g} / \mathrm{dl}$ ), in addition to the following: Red blood count, $3.89 \times 10^{12}$ cells $/ 1$ (reference, $4.3-5.8 \times 10^{12}$ cells/1 ); mean corpuscular volume, $65.8 \mathrm{fl}$ (reference, 82-100 fl); mean hemoglobin content, $18 \mathrm{pg}$ (reference, 27-34 pg); mean hemoglobin concentration, $270 \mathrm{~g} / 1$ (reference, 316-354 g/l); and white blood cell (WBC) count, $8.91 \times 10^{9}$ cells/ 1 (reference, 3.5-9.5 $\times 10^{9}$ cells/1. Serum tumor marker levels were: Cancer antigen-125, 68.3 U/ml (reference, 0-35 U/ml), Cyfra21-1, $4.19 \mathrm{ng} / \mathrm{ml}$ (reference, 0-3.3 ng/ml); and neuron-specific enolase, $49.78 \mathrm{ng} / \mathrm{ml}$ (reference, 0-17 ng/ml). Abnormal thyroid function was detected: Serum total thyroxine (T4), $6.65 \mu \mathrm{g} / \mathrm{dl}$ (reference, 5.1-14.1 $\mu \mathrm{g} / \mathrm{dl}$ ); total triiodothyronine (T3), $85.3 \mathrm{ng} / \mathrm{dl}$ (reference, 80-200 ng/dl); thyrotropin, $9.68 \mathrm{IU} / \mathrm{ml}$ (reference, $0.27-4.2 \mathrm{~g} / \mathrm{dl}$ ); free $\mathrm{T} 3,1.67 \mathrm{pg} / \mathrm{ml}$ (reference, 2-4.4 pg/ml); and free T4, $0.94 \mathrm{ng} / \mathrm{dl}$ (reference, 0.93-1.7 ng/dl).

Ultrasound investigation (ACUSON Sequoia 512; Siemens Healthineers, Erlangen, Germany) indicated that the right-hand side of the patient's neck was hyperechoic and computed tomography (CT) of the chest (Somatom Definition AS; Siemens Healthineers) demonstrated a mass in the upper lobe of the right lung, multiple nodules in both lungs and pleural effusion in the right lung (Fig. 2).

The initial diagnosis was right upper pulmonary neoplasm with right cervical lymph node and pleural metastases; therefore, an ultrasound-guided right cervical mass biopsy was performed. Histopathological analysis of the aspiration tissue (Santa Cruz Biotechnology, Inc., La Jolla, TX, USA) demonstrated granulation tissue and abscess formation (Fig. 3A). A modified acid-fast stain-positive organism was detected with smooth and granular colonies (Fig. 3B and C). Subculture and microscopy demonstrated the organism was a branching gram-positive rod; thus, Nocardia was confirmed. The cutaneous, soft tissue lesion and lung symptoms were diagnosed as nocardiosis.

As the patient presented with nocardiosis with the characteristics of dissemination, further examinations were performed. Firstly, routine and chemical testing of pleural effusion was performed, demonstrating the effusion was $\mathrm{pH}$ 7.4, and positive for Rey's reaction, which indicates exudate. The proportion of pleural fluid was 1.025, and the WBC count was significantly elevated at $1.8 \times 10^{9}$ cells $/ 1$, with $91 \%$ monocytes and $9 \%$ polymorphonuclear cells. Protein concentration of the hydrothorax was $32.5 \mathrm{~g} / \mathrm{l}$, and the concentrations of glucose, lactate dehydrogenase and adenosine deaminase were $2.1 \mathrm{mmol} / 1,468 \mathrm{U} / 1$ and $27 \mathrm{U} / 1$, respectively. These results confirmed a parapneumonic effusion. Contrast-enhanced CT of the abdomen indicated multiple low-density lesions in the right lobe of the liver, which, with ring-enhancement, were consistent with the imaging features of an abscess (Fig. 4). MRI scanning of the head (Magnetom Avanto 1.5T; Siemens

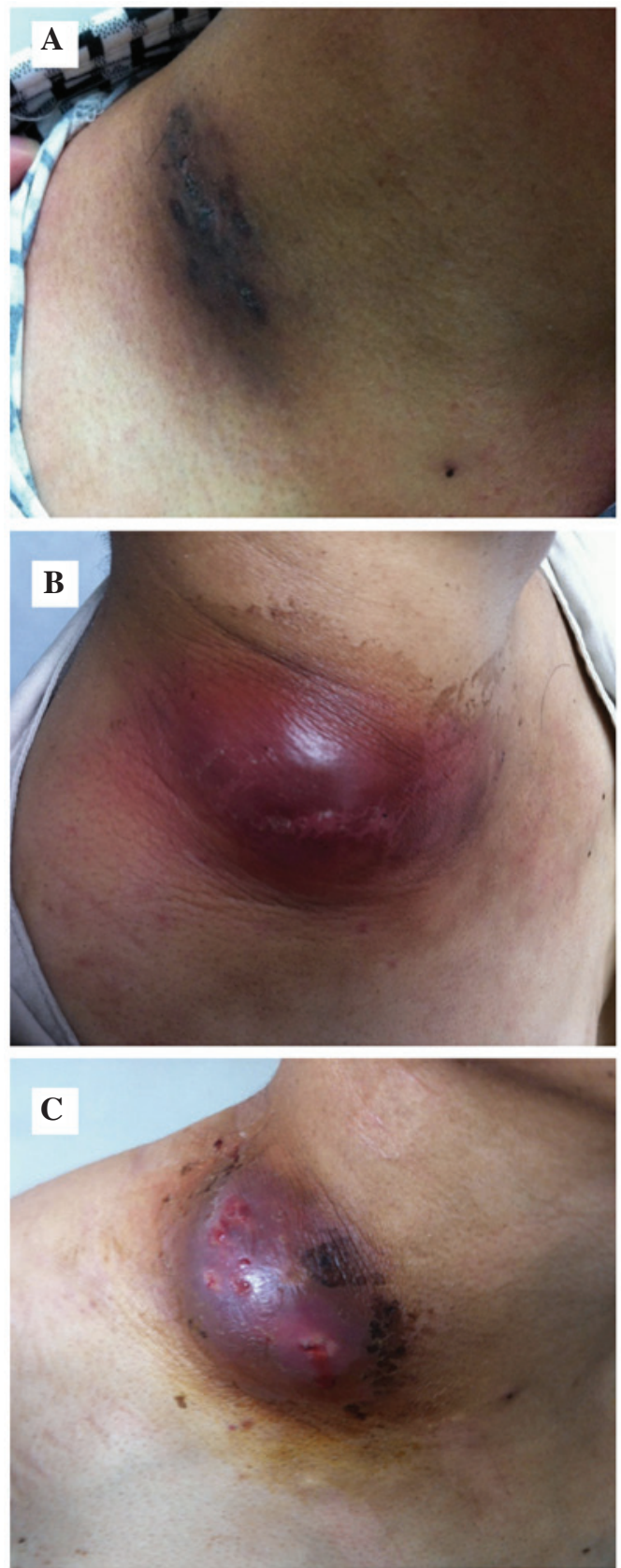

Figure 1. Visual observation of the patient's neck. (A) Mass on the right side of the patient's neck (size, $12 \times 8 \mathrm{~cm}$ ). After treatment for (B) 20 days and (C) 2 months.

Healthineers) demonstrated no abnormalities. Therefore the patient was diagnosed with disseminated nocardiosis of the subcutaneous soft tissue, lungs and liver.

The patient was initially treated with ceftriaxone ( $2 \mathrm{~g} /$ day) and sulfamethoxazole trimethoprim (2 tablets twice daily). Levothyroxine sodium ( $25 \mu \mathrm{g} / \mathrm{day})$ was administered for subclinical hypothyroidism. Since the diagnosis of disseminated nocardiosis was confirmed, etiological investigations and drug sensitivity testing was conducted. Culturing of the mass biopsy tissue showed that the patient was positive for $N$. otitidiscaviarum. Using the disk diffusion method (Kirkby-Bauer method), susceptibility testing demonstrated the following diameters for areas of non-bacterial growth in response to various antibiotics: Amoxicillin-clavulanic 

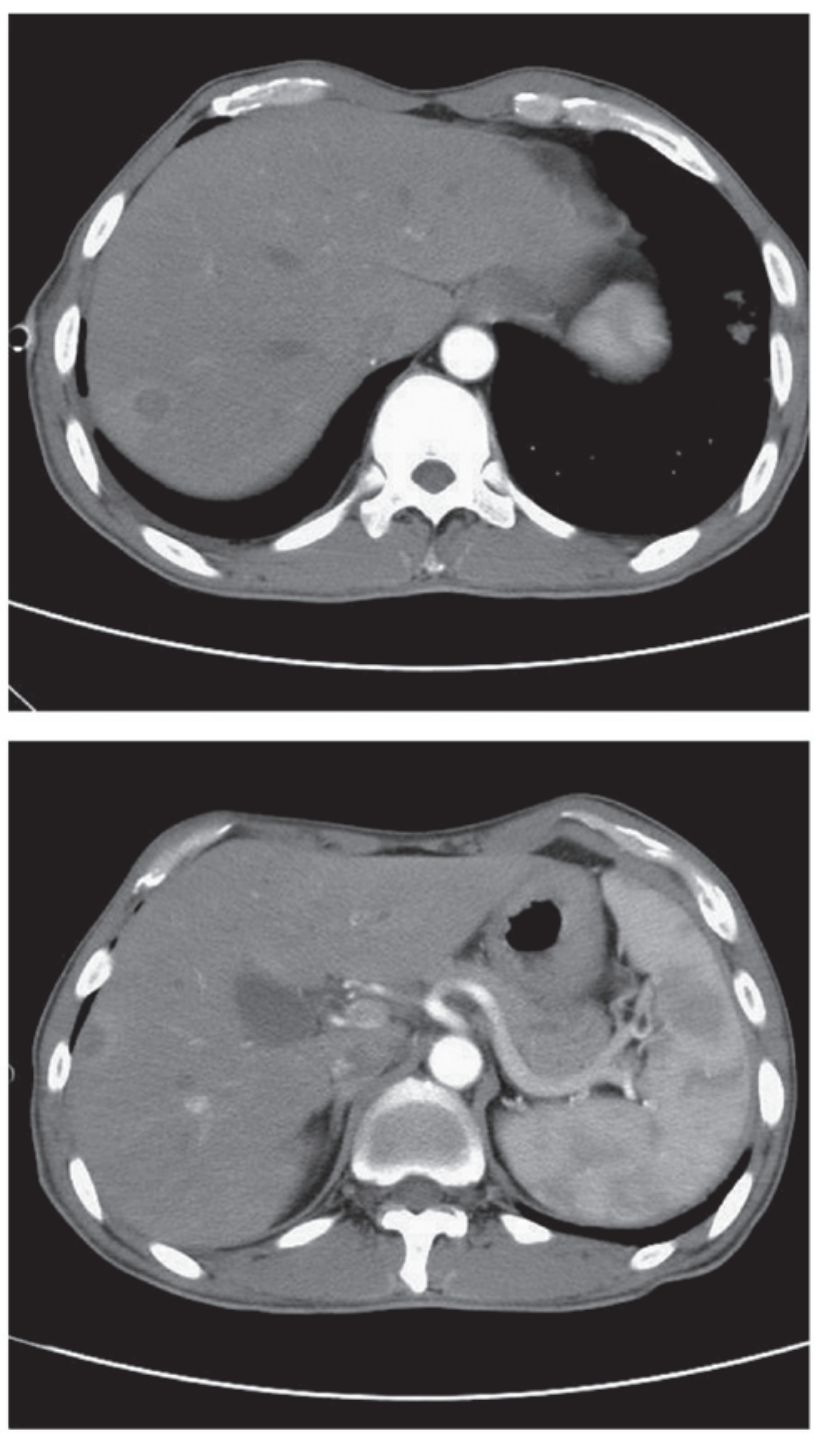

Figure 2. Chest computed tomography showed consolidation in the upper lobe of right lung, multiple nodules in both lung and right pleural effusion.

acid, $6 \mathrm{~mm}$; cefepime $6 \mathrm{~mm}$; imipenem, $6 \mathrm{~mm}$; cefotaxime, $6 \mathrm{~mm}$; ceftriaxone, $9 \mathrm{~mm}$; erythromycin, $12 \mathrm{~mm}$; ampicillin, $16 \mathrm{~mm}$; gentamicin, $22 \mathrm{~mm}$; ciprofloxacin, $24 \mathrm{~mm}$; trimethoprim-sulfamethoxazole (TMP-SMZ), $30 \mathrm{~mm}$; linezolid, $38 \mathrm{~mm}$; minocycline, $40 \mathrm{~mm}$; and amikacin, $42 \mathrm{~mm}$. Therefore, on the basis of these results, combined minocycline and TMP-SMZ therapy was chosen. Following administration for 2 months, the mass on the right-hand side of the patient's neck markedly reduced in size (Fig. 1B and C) and the lung lesion gradually improved.

\section{Discussion}

The PubMed database was searched and 23 cases were retrieved with 'Nocardia otitidiscaviarum' as the search term from January 1990 to August 2014 as shown in Table I (3-25). These 23 cases included 11 cases of subcutaneous and soft tissue involvement, 5 patients with pulmonary involvement, 2 cases of brain involvement, 1 case of pyothorax involvement, 1 case of subcutaneous, soft tissue and lung involvement, 1 case of subcutaneous, soft tissue and brain involvement, 1 case of
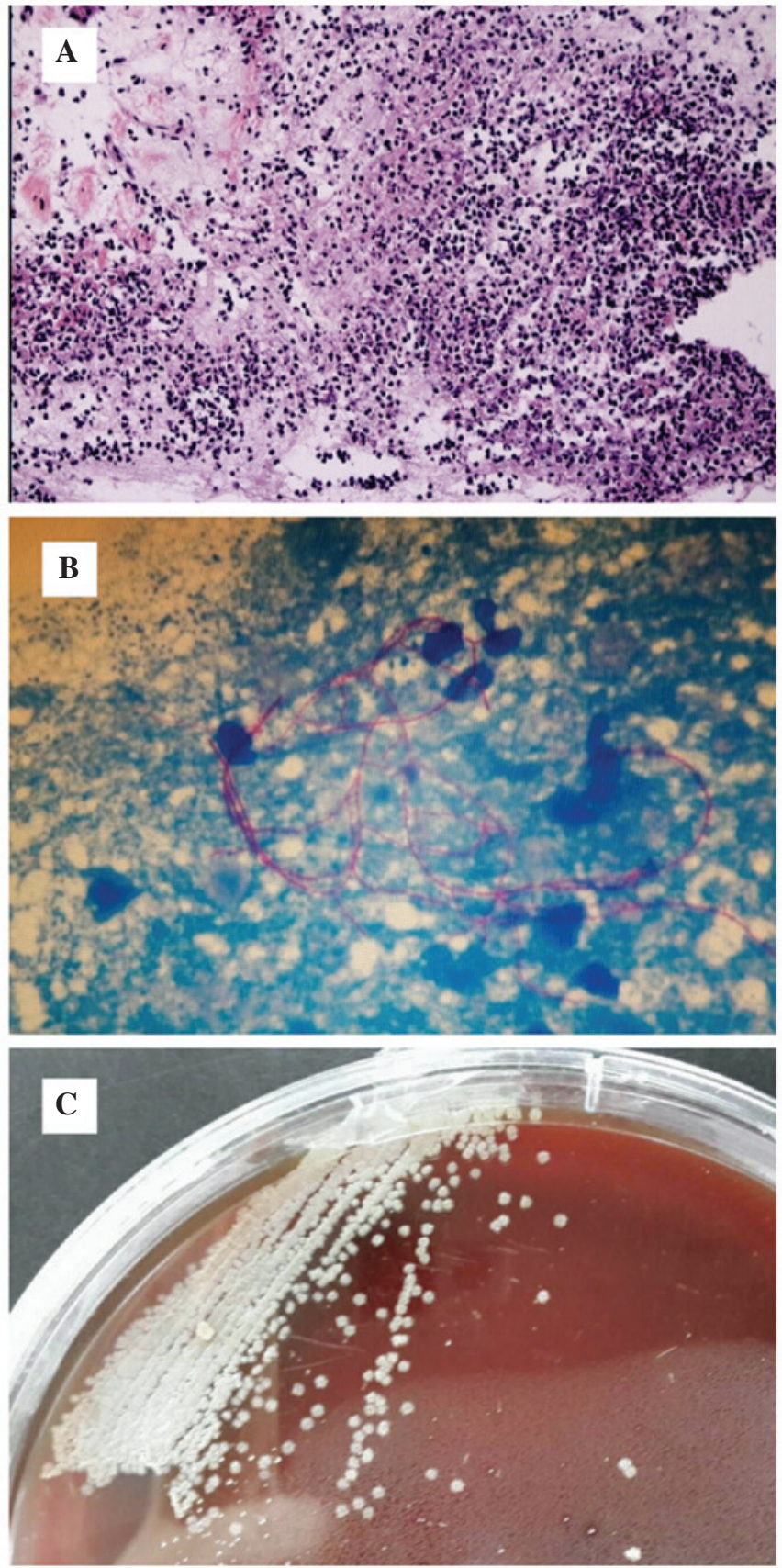

Figure 3. (A) Low power microscopy demonstrated a large amount of necrotic neutrophils, which indicated a purulent lesion. (B) Nocardia growth was confirmed by microscopy for a branching gram-positive rod, which was demonstrated to be positive for modified acid-fast stain. (C) Nocardia growth was observed by subculture, which demonstrated smooth and granular colonies.

pyothorax and brain involvement, and 1 case with chest wall abscesses, bilateral pulmonary infiltrates and intra-abdominal mass involvement. The mean age of the patients was $56.61 \pm 18.58$ years, and there were 6 female and 17 male patients. Nine cases were immunocompetent patients, of which 7 cases presented with cutaneous infection; all but one of the patients with cutaneous infection had a history of trauma. The remaining two cases presented with lung infections with no history of trauma. A total of 14 cases were immunosuppressed patients, including the following conditions: Rheumatoid joints $(n=3)$; acquired immune deficiency syndrome (AIDS; $n=2)$; intravenous drug use $(n=1)$; renal transplantation $(n=1)$; 


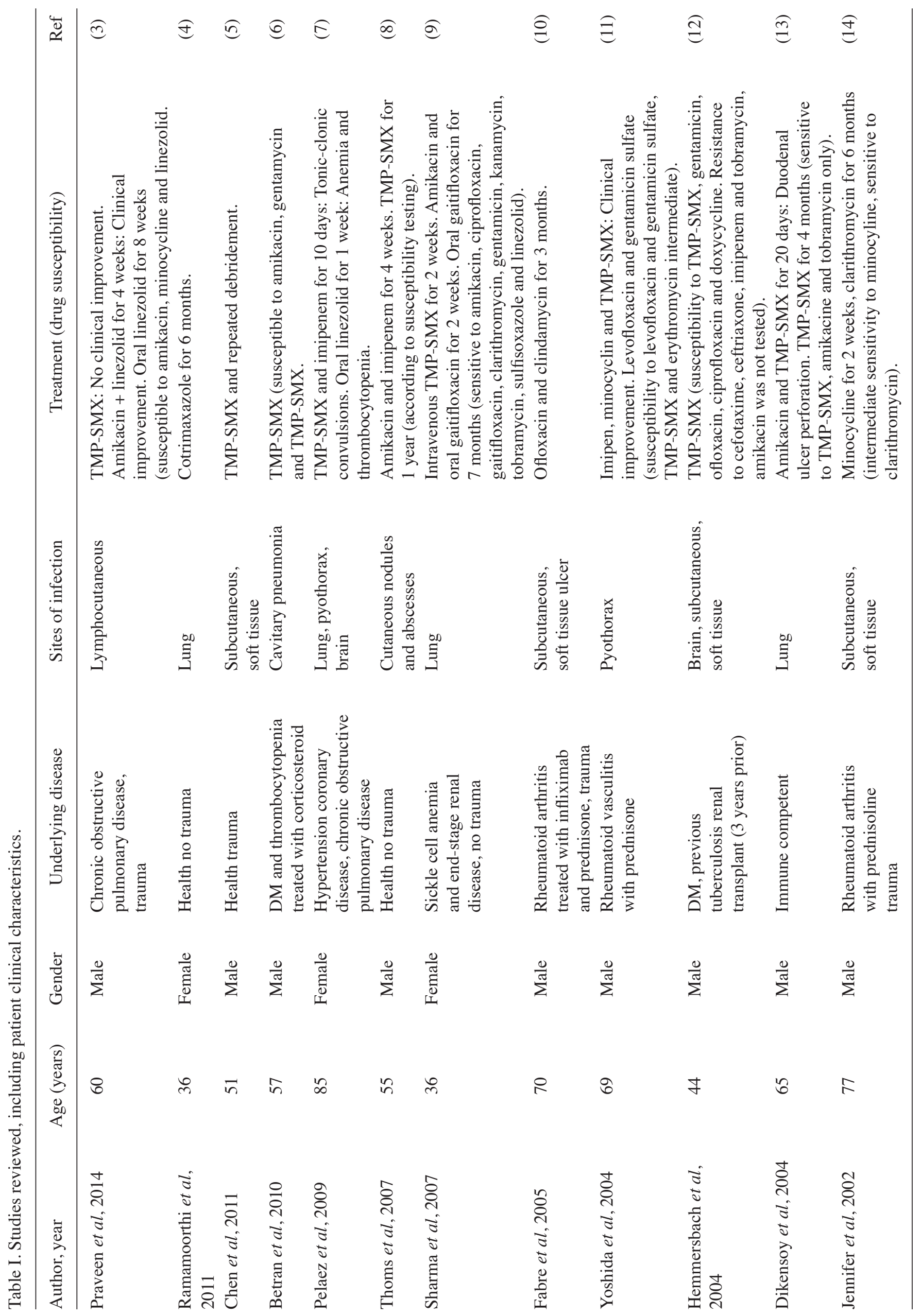




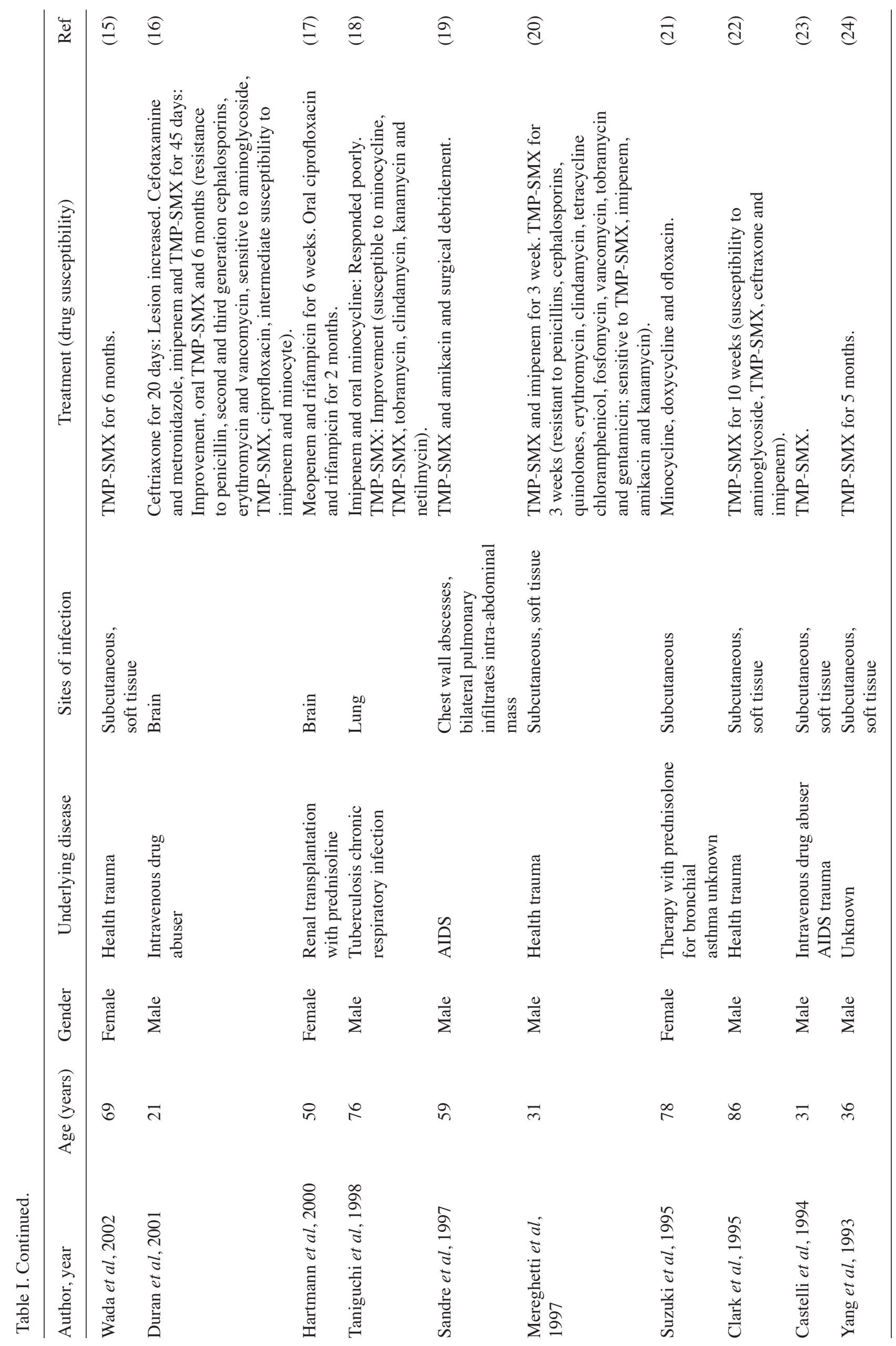


heart transplantation $(\mathrm{n}=1)$; asthma $(\mathrm{n}=1)$; chronic obstructive pulmonary disease $(\mathrm{n}=1)$; diabetes mellitus and thrombocytopenia $(\mathrm{n}=1)$; sickle cell anemia and chronic renal insufficiency $(\mathrm{n}=1)$; diabetes mellitus and renal transplantation $(\mathrm{n}=1)$; and tuberculosis and chronic respiratory infection $(n=1)$. Among these immunosuppressed patients, 9 patients were treated with corticosteroids (9/14); the exceptions were 2 cases of AIDS, 1 case of intravenous drug abuse, 1 case of sickle cell anemia and chronic renal insufficiency and 1 case of tuberculosis and chronic respiratory infection. Among the 23 cases, 13 patients underwent drug susceptibility testing, which demonstrated that $77 \%$ of cases (10/13) were sensitive to TMP-SMX, with the remaining 3 cases being resistant to TMP-SMX. Furthermore, 12 cases (12/13) were sensitive to aminoglycoside and 12 cases $(12 / 13)$ were resistant to cephalosporin.

According to the site of infection, human nocardiosis can be clinically classified into nocardial mycetoma or pulmonary, extrapulmonary, central nervous system, cutaneous or subcutaneous nocardiosis (3).

The present case is a patient who initially presented with a persistent cough (lasting 50 days) followed by a right cervical mass with no history of trauma. The diagnosis was primary lung infection caused by $N$. otitidiscaviarum. The patient had participated in carpentry work for 3 years and often had contact with soil and sawdust, and the infection may have been introduced into the respiratory tract via the inspiration of soil and/or sawdust.

Pulmonary nocardiosis is often associated with nocardia empyema, and it has been reported that $N$. otitidiscaviarum is capable of inducing empyema (11). Analysis of the right pleural effusion in the present case demonstrated that it was an exudate dominated by monocytes ( $\mathrm{pH} 7.4$ ) with $2.1 \mathrm{mmol} / 1$ glucose. These results indicated that the pleural fluid was parapneumonic without empyema.

It has previously been reported that Nocardia spp. are capable of causing disseminated disease that can readily enter the bloodstream and spread throughout the body, including the skin, lung, central nervous system and abdominal organs (3). Therefore, contrast-enhanced CT was performed, which detected a liver abscess, and cranial MRI demonstrated no structural disease. These results confirmed the diagnosis of disseminated nocardiosis of the subcutaneous soft tissue, lungs and liver.

N.otitidiscaviarum was first isolated in 1924 by Snijders (26). Gordon and Mihm were the first to define the biochemical criterion for its identification and distinguish it from other Nocardia species (27). Although the name Nocardia caviae was originally proposed, the organism is currently known as N.otitidiscaviarum, as defined in Bergey's Manual of Systematic Bacteriology (28). Although primary nocardiosis may be induced by infection with any Nocardia spp., N. brasiliensis is isolated from the majority of cases $(\sim 80 \%)$ (29). In a study conducted in the USA, only $2.9 \%$ (10/347) of infections due to Nocardia were identified as N. otitidiscaviarum (30). In Germany, Nocardia spp. were isolated in 131 patients at a national reference laboratory between 1979 and 1991, with only 8 patients having $N$. otitidiscaviarum infections (31). Therefore $N$. otitidiscaviarum causes fewer infections than other species of Nocardia. This low incidence has been attributed to reduced pathogenicity or its lower prevalence in soil (32). 

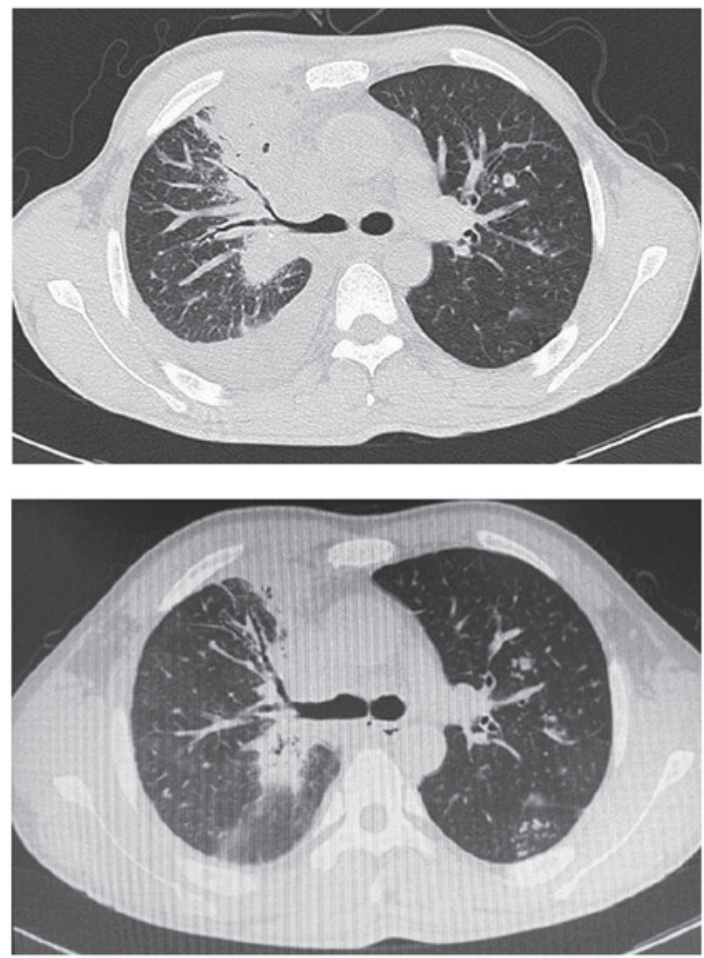
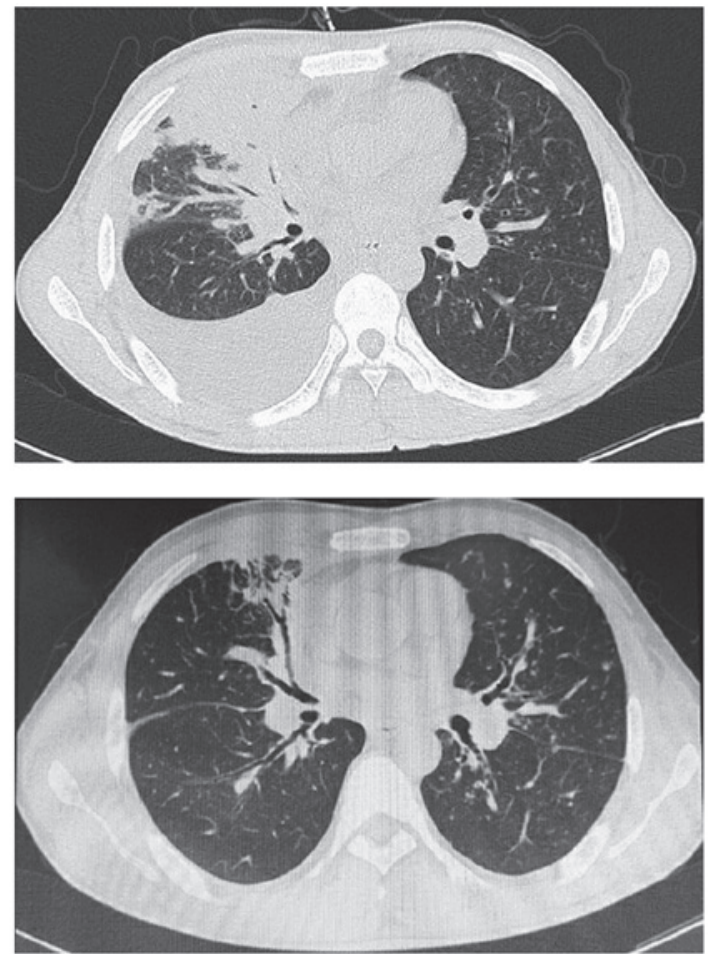

Figure 4. Enhanced computed tomography of the patient's abdomen demonstrated multiple low-density lesions in the right lobe of the liver, which suggested the presence of an abscess.

Tan et al (33) retrospectively reviewed the laboratory records of the Bacteriology Laboratory at National Taiwan University Hospital between January 1998 and June 2008 and discovered that the major types of Nocardia infection were cutaneous infection (56.6\%), pulmonary infection (33.6\%) and disseminated infection (7.1\%). Therefore, disseminated nocardiosis is a rare disease. It has also been demonstrated that disseminated systemic infection arises in the respiratory tract and is usually associated with $N$. asteroides, in both healthy and immunocompromised hosts, although immunocompromised patients have a greater tendency to develop pulmonary or disseminated disease (34). The present patient was a healthy host with disseminated nocardiosis caused by $N$. otitidiscaviarum, which is rare. To the best of our knowledge, the present case report is the first to describe a case of disseminated nocardiosis of the subcutaneous soft tissue, lungs and liver, caused by N. otitidiscaviarum in an immunocompetent host.

The present report is also the first to summarize the literature concerning the clinical characteristics of N. otitidiscaviarum from 1990 to 2014, to the best of our knowledge. The present literature review demonstrated that subcutaneous and soft tissue were the most common sites of infection with $N$. otitidiscaviarum (14/23 cases). Amongst the 23 cases retrieved, $39 \%$ of the patients were immunocompetent (9/23). The majority of cases of primary cutaneous nocardiosis in healthy patients had a history of local trauma (5/9 cases). Three immunocompetent patients presented with primary lung infection with no history of trauma, and may have become infected by exposure to soil, although $N$. otitidiscaviarum has a lower prevalence in soil, as compared with other Nocardia spp. These 3 cases involved only the lungs, and were not disseminated infections; therefore, the present patient is the only case of disseminated infection caused by $N$. otitidiscaviarum in a healthy patient without a history of local trauma, to date.

Glucocorticoids are well characterized and widely used for immune suppression, however, glucocorticoid use is also one of the risk factors for invasive nocardiosis $(1,11)$. In the present review, corticosteroid administration was considered to be a major contributors to the impaired immunity of the patients (9/23 cases).

Nocardia spp. infection can be observed and confirmed by subculture and positive microscopic detection of a branching gram-positive rod and modified acid-fast stain. It is clinically imperative that the species be identified, as $N$. farcinica is resistant to third generation cephalosporins and tobramycin, and $N$. brasiliensis is resistant to minocycline (35). Although there is no uniform standard or recommended application method from the National Committee for Standardization of Clinical Trials, the majority of previous studies have used the diameter of a zone of inhibition to determine antimicrobial susceptibility (36). It has previously been reported that the sensitivity of $N$. otitidiscaviarum to $\beta$-lactam antibiotics is poor (37), which is consistent with the results of the present case. According to the economic conditions of the patient, minocycline combined with TMP-SMZ was recommended.

As with other types of nocardial infections, the optimal duration of antibiotic therapy for $N$. otitidiscaviarum infections remains unknown. Brown-Elliott et al (29) previously reported that relapse was rare in cases treated for 4-6 months, although prolonged antibiotic chemotherapy may be required for complete eradication. Furthermore, it has been reported that systemic symptoms disappear after $\geq 6$ weeks, and that immunosuppressed patients should be treated for $\geq 6$ months and patients with central nervous system involvement should 
sustain treatment for 12 months (9). In nocardiosis cases with CNS involvement, treatment with a combination of antibiotics, such as amikacin and minocycline, is recommended for $\geq 2$ months (13). Although treatment with sulfonamides has been effective in the majority of cases, susceptibility testing for individual patients should guide definitive therapy, particularly for $N$. otitidiscaviarum infections, which may be resistant to these agents $(10,11,19)$. The present patient received minocycline and TMP-SMZ for 4 months and was considered to be cured.

In conclusion, the present study is the first report of disseminated $N$. otitidiscaviarum infection in an immunocompetent patient without a history of trauma. Clinical and microbiological manifestations of nocardiosis vary for different Nocardia spp.; therefore accurate identification of the species is crucial for effective diagnosis and treatment. Susceptibility testing should guide definitive therapy, particularly for N. otitidiscaviarum infections, which may be resistant to $\beta$-lactam antibiotics. Further experience in the treatment of $N$. otitidiscaviarum infections is required in order to assess drug susceptibility and clinical outcomes.

\section{References}

1. Faghri J, Bourbour S, Moghim S, Meidani M, Safaei HG, Hosseini N, Esfahani BN, Fazeli $\mathrm{H}$ and Sedighi M: Comparison of three phenotypic and deoxyribonucleic acid extraction methods for isolation and identification of Nocardia spp. Adv Biomed Res 3: 151-154, 2014.

2. Yaşar Z, Acat M, Onaran H, Ozgü MA, Fener N, Talay F and Cetinkaya E: An unusual case of pulmonary nocardiosis in immunocompetent patient. Case Rep Pulmonol 2014: 963482, 2014.

3. Shahapur PR, Peerapur BV, Shahapur RP, Honnutagi RM and Biradar MS: Lymphocutaneous nocardiosis caused by Nocardia otitidiscaviarum. A case report and review of literature. J Nat Sci Biol Med 5: 197-201, 2014.

4. Ramamoorthi K, Pruthvi BC, Rao NR, Belle J and Chawla K: Pulmonary nocardiosis due to Nocardia otitidiscaviarum in an immunocompetent host - a rare case report. Asian Pac J Trop Med 4: 414-416, 2011.

5. Chen B, Zhu LY, Xuan X, Wu LJ, Zhou TL, Zhang XQ and Li BX: Isolation of both Pseudozyma aphidis and Nocardia otitidiscaviarum from a mycetoma on the leg. Int J Dermatol 50: 714-719, 2011.

6. Betrán A, Villuendas MC, Rezusta A, Moles B, Rubio MC, Revillo MJ, Boiron P, Bello S and Rodríguez-Nava V: Cavitary pneumonia caused by Nocardia otitidiscaviarum. Braz J Microbiol 41: 329-332, 2010.

7. Pelaez AI, Garcia-Suarez MM, Manteca A, Melon O, Aranaz C, Cimadevilla R, Mendez FJ and Vazquez F: A fatal case of Nocardia otitidiscaviarum pulmonary infection and brain abscess: Taxonomic characterization by molecular techniques Ann Clin Microbiol Antimicrob 8: 11-16, 2009.

8. Thoms KM, Zimmermann O, Schupp P, Thoms S and Emmert S: Nocardia otitidiscaviarum: Cause of long-term cutaneous abscesses on the leg of an immunocompetent man. Arch Dermatol 143: 1086-1087, 2007.

9. Sharma M, Gilbert BC, Benz RL and Santoro J: Disseminated Nocardia otitidiscaviarum infection in a woman with sickle cell anemia and end-stage renal disease. Am J Med Sci 333: 372-375, 2007.

10. Fabre S, Gibert C, Lechiche C, Jorgensen C and Sany J: Primary cutaneous Nocardia otitidiscaviarum infection in a patient with rheumatoid arthritis treated with infliximab. J Rheumatol 32: 2432-2433, 2005.

11. Yoshida K, Bandoh S, Fujita J, Tokuda M, Negayama K and Ishida T: Pyothorax caused by Nocardia otitidiscaviarum in a patient with rheumatoid vasculitis. Intern Med 43: 615-619, 2004.

12. Hemmersbach-Miller M, Martel AC, Benítez AB and Sosa AO: Brain abscess due to Nocardia otitidiscaviarum: Report of a case and review. Scand J Infect Dis 36: 381-384, 2004.

13. Dikensoy O, Filiz A, Bayram N, Balci I, Zer Y, Celik G and Ekinci E: First report of pulmonary Nocardia otitidiscaviarum infection in an immunocompetent patient from Turkey. Int J Clin Pract 58: 210-213, 2004.
14. Alberts JH and Boyd AS: Nocardia otitidiscaviarum: An unusual Nocardia species causing a primary lymphocutaneous infectious process in a mildly immunosuppressed patient. Skinmed 1: 62-64, 2002.

15. Wada A, Matsuda S, Kubota H, Miura H and Iwamoto Y: Primary lymphocutaneous nocardiosis caused by Nocardia otitidiscaviarum. Hand Surg 7: 285-287, 2002.

16. Durán E, López L, Martínez A, Comuñas F, Boiron P and Rubio MC: Primary brain abscess with Nocardia otitidiscaviarum in an intravenous drug abuser. J Med Microbiol 50: 101-103, 2001.

17. Hartmann A, Halvorsen CE, Jenssen T, Bjørneklett A, Brekke IB, Bakke SJ, Hirschberg H, Tønjum T and Gaustad P: Intracerebral abscess caused by Nocardia otitidiscaviarum in a renal transplant patient cured by evacuation plus antibiotic therapy. Nephron 86: 79-83, 2000.

18. Taniguchi H, Mukae H, Ashitani J, Ihi T, Sakamoto A, Kohno S and Matsukura S: Pulmonary Nocardia otitidiscaviarum infection in a patient with chronic respiratory infection. Intern Med 37: 872-876, 1998.

19. Sandre RM and Summerbell RC: Disseminated Nocardia otitidiscaviarum in a patient with AIDS. Can J Infect Dis 8: 347-350, 1997.

20. Mereghetti L, van der Mee-Marquet N, Dubost AF and Boiron P: Nocardia otitidiscaviarum infection of a traumatic skin wound. Eur J Clin Microbiol Infect Dis 16: 383-384, 1997.

21. Suzuki Y, Toyama K, Utsugi K, Yazawa K, Mikami Y, Fujita M and Shinkai H: Primary lymphocutaneous nocardiosis due to Nocardia otitidiscaviarum: The first case report from Japan. J Dermatol 22: 344-347, 1995.

22. Clark NM, Braun DK, Pasternak A and Chenoweth CE: Primary cutaneous Nocardia otitidiscaviarum infection: case report and review. Clin Infect Dis 20: 1266-1270, 1995.

23. Castelli L, Zlotnik H, Ponti R and Vidotto V: First reported Nocardia otitidiscaviarum infection in an AIDS patient in Italy. Mycopathologia 126: 131-136,1994.

24. Yang LJ, Chan HL, Chen WJ and Kuo TT: Lymphocutaneous nocardiosis caused by Nocardia caviae: The first case report from Asia. J Am Acad Dermatol 29: 639-41,1993.

25. Simmons BP, Gelfand MS and Roberts GD: Nocardia otitidiscaviarum (caviae) infection in a heart transplant patient presented as having a thigh abscess (Madura thigh). J Heart Lung Transplant 11: 824-826, 1992.

26. Snijders EP: Verslag van het wetenschappenllijk gdeelte der vergaderingen van der afdelling Sumatra's oostkurst. Geneesk Tijdschr Ned Indie 64: 75-77, 1924 (In Dutch).

27. Ferreira NP and Tracey RP: Numerical taxonomy of cholesterol-degrading soil bacteria. J Appl Microbiol 57: 429-446, 2008

28. Goodfellow M, Kämpfer P, Busse HJ, Trujillo ME, Suzuki KI, Ludwig W and Whitman WB (eds): The Actinobacteria. In: Bergey's Manual of Systematic Bacteriology. Vol 5. 2nd edition. Springer, New York, NY, 2012.

29. Gomez-Flores A, Welsh O, Said-Fernández S, Lozano-Garza G, Tavar-Alejandro RE and Vera-Cabrera L: In Vitro and In Vivo Activities of Antimicrobials against Nocardia brasiliensis. Antimicrob Agents Chemother 48: 832-837, 2004.

30. Brown-Elliott BA, Brown JM, Conville PS and Wallace RJ Jr: Clinical and laboratory features of the Nocardia spp. based on current molecular taxonomy. Clin Microbiol Rev 19: 259-282, 2006.

31. Schaal KP and Lee HJ: Actinomycete infections in humans: A review. Gene 115: 201-211, 1992.

32. Duran E, Lopez L, Martinez A, Comuñas F, Boiron P and Rubio MC: Primary brain abscess with Nocardia otitidiscaviarum in an intravenous drug abuser. J Med Microbiol 50: 101-103, 2001.

33. Tan CK, Lai CC and Lin SH: Clinical and microbiological characteristics of Nocardiosis including those caused by emerging Nocardia species in Taiwan, 1998-2008. Clin Microbiol Infect 16: 966-972, 2010

34. Mari B, Montón C and Mariscal D: Pulmonary nocardiosis: Clinical experience in ten cases. Respiration 68: 382-388, 2001.

35. Miralles GD: Disseminated Nocardia farcinica infection in an AIDS patient. Eur J Clin Microbiol Infect Dis 13: 497-500, 1994.

36. McNeil MM and Brown JM: The medically important aerobic actinomycetes: Epidemiology and microbiology. Clin Micmbiol Rev 7: 357-417, 1994.

37. Zhang W, Peng GJ, Liu J, $\mathrm{Hu} \mathrm{H}$ and Liang $\mathrm{H}$ : Identification of rare Nocardia otitidiscaviarum and its drug sensitivity analysis. Int J Lab Med 35: 1320-1325, 2014. 